\title{
Erratum to: Different thermal conductivity in drilling of cemented compared with cementless hip prostheses in the treatment of periprosthetic fractures of the proximal femur-an experimental biomechanical analysis
}

\author{
Stephan Brand ${ }^{1}$ - Johannes $\mathrm{Klotz}^{2} \cdot$ Thomas Hassel $^{2} \cdot$ Maximilian Petri $^{1}$ • \\ Max Ettinger $^{3} \cdot$ Christian Krettek $^{1} \cdot$ Thomas Goesling $^{1} \cdot$ Friedrich-Wilhelm Bach $^{2}$
}

Erratum to: International Orthopaedics (SICOT) (2013) 37:1885-1889

DOI 10.1007/s00264-013-1964-8

The original version of this article contained mistake. The correct Figure legends for Figures 1, 3 and 5 should have been the following:

Fig. 1. HPC (high performance cutting) drill with internally applied cooling system via cooling channels through the tip $\left(\sigma=140^{\circ} ;\right.$ Perschmann $)[6]$
Fig. 3. Temperature changes during drilling of cemented implants with an internally cooled HPC (high performance cutting) drill $(\mathrm{D}=5.5 \mathrm{~mm})[6]$

Fig. 5. Thermographic scan of drilling procedure [6]

Corresponding reference is:

6. Brand S, Klotz J, Petri M, Ettinger M, Hassel T, Krettek C, Goesling T, Bach FW (2013) Temperature control with internally applied cooling in solid material drilling: an experimental, biomechanical study. Int Orthop. Mar 20 [Epub ahead of print]. doi:10.1007/s00264-013-1850-4

The online version of the original article can be found at http://dx.doi.org/ 10.1007/s00264-013-1964-8.

Stephan Brand

brand.stephan@mh-hannover.de

Trauma Department, Hannover Medical School, MHH, Carl-Neuberg-Str. 1, 30625 Hannover, Germany

2 Institute of Material Sciences, Leibniz University Hannover, Hannover, Germany

3 Department of Orthopedic Surgery, Hannover Medical School, Hannover, Germany 